\title{
Computer Modelling of Sound Propagation in Enclosed Spaces with Uneven Fitting Distributions
}

\author{
S.M. Dance and B.M. Shield \\ The Acoustics Group, School of Engineering Systems and Design, South Bank University, London SE1 OAA, UK
}

\begin{abstract}
Current image-source based computer models assume an even floor distribution of fittings in non-diffuse enclosed spaces, such as factories. This is not fully representative of industrial spaces where gangways and open areas are present. It has been shown that the more precise the representation of the fittings the greater the prediction accuracy of the steady state sound levels. This paper presents a validation of the Complete Image Source Model (CISM) in two test spaces, a 1:10 scale model and a laboratory space configured with six different fitting distributions, which previously could not be modelled by image-source based methods. Sound propagation curves were predicted for each fitting configuration, and are compared with sound propagation curves given by a version of the Ondet and Barbry ray-tracing model, RAYCUB. The sound levels predicted by RAYCUB and CISM were found to be of a similar accuracy in all six cases, but CISM required less information to define the space and less time to produce the results.
\end{abstract}

\section{INTRODUCTION}

The accurate prediction of steady state sound distributions in factory spaces where machinery or fittings are not evenly distributed over the shop floor has not previously been possible using the image-source method. It has been shown that an accurate representation of the positions of groupings of fittings in a model is of critical importance. ${ }^{1}$ The unevenness of the distribution is typically caused by gangways and open areas in industrial spaces.

Current computer prediction models have been validated in either test spaces, where the fittings were distributed isotropically ${ }^{2}$ or uniformly across the entire floor area. ${ }^{3}$ The first validated model capable of representing arbitrary fitting distributions was RAYCUB by Ondet and Barbry and hence is used for comparative purposes in this investigation in an enhanced form. ${ }^{4}$ Although none of the enhancements were used in the cases detailed below, except shortened run time lengths approximately one-fifth of the time previously required.

This paper describes the validation of the image-source model, CISM, in two simplified test spaces, a laboratory space and a physical scale model. The two test spaces were each configured with uneven-fitting distributions in three different ways, giving a total of six sets of validation data. The validation results are presented in the form of sound propagation curves and result tables consisting of average prediction difference, data size and run-time length.

\section{THE PREDICTION MODELS}

The two computer models CISM and RAYCUB, which have been developed, based on geometric acoustics, for the prediction of sound distribution in non-diffuse fitted enclosed spaces, are described below.

\subsection{The CISM Model}

CISM, developed by Dance and Shield ${ }^{5}$ is based on the image-source method of geometric computer modelling rather than the ray-tracing technique used by RAYCUB.
However, the development of CISM was guided by those parameters found to be of critical importance to the RAYCUB model when predicting the sound levels in factory spaces and hence is applicable to steady state predictions in the $125 \mathrm{~Hz}$ to $4 \mathrm{kHz}$ frequency range. Each frequency must be predicted individually, although an approximate $\mathrm{dB}(\mathrm{Lin})$ prediction has been found to be accurate. ${ }^{1}$

The model assumes the space to be a parallelepiped with absorption coefficients, which are defined as independent of the angle of incidence, for each room surface. Arbitrarily positioned rectangular absorptive patches can be defined on each room surface, instead of dividing the surface into rectangular areas of different absorption coefficients, as in ray-tracing models. Additional totally-sound-absorbing surfaces can be installed in the room if they are parallel to one of the room surfaces and as such can be used to represent acoustic barriers. Sound sources are treated as point sources with associated sound power and directivity characteristics.

Fittings in CISM are represented statistically using the Kuttruff equation for scattering frequency. ${ }^{6}$ The volume of the space can be divided or zoned in three ways: isotropic zoning, where the statistical fittings are distributed evenly around the entire volume; secondly, floor zoning, where the room is divided by an acoustically transparent plane into two, with a zonal volume of fittings near the floor and usually an empty zone above; and thirdly, gangway zoning, where the room is divided into three horizontal or vertical zonal volumes. Each zone may be treated as either fitted or empty, if fitted a scattering frequency is used to represent the statistical density of the fittings and an absorption coefficient is required for the objects inside the volume. When the sound is inside the zone, it is attenuated by a factor $F$ given by

$$
F_{i, j, k}=(1-a)^{d_{i, j, k} q},
$$

where $\alpha \mathrm{a}$ is the absorption coefficient of the fittings in the zone, $d$ is the distance travelled by the sound inside the zone, and $q$ is the scattering frequency of the zone. The subscripts $i, j, k$ denote the number of reflections from the room surfaces in each direction $x, y$ and $z$, for each unique sound path. 